\title{
Video record (CD copy attached) of the Spain bolide of June 14, 1996: The atmospheric trajectory and orbit
}

\author{
J.A. Docobo ${ }^{1}$ and Z. Ceplecha ${ }^{2}$ \\ 1 Astronomical Observatory "Ramon Maria Aller", University of Santiago de Compostela, Spain \\ e-mail: oadoco@usc.es \\ 2 Astronomical Institute of the Academy of Sciences, 25165 Ondřejov Observatory, Czech Republic \\ e-mail: ceplecha@asu.cas.cz
}

Received November 26, 1998; accepted February 2, 1999

\begin{abstract}
On June 14, 1996, a very bright bolide (SP960614) appeared over Galicia, the NW region of Spain. A casual video record of the bolide flight is available and its CD ROM copy included in this paper. We were able to deduce accurate horizontal coordinates from digitized video-recorded images and combine them with numerous visual observations. In order to get reasonable data from visual sightings, we visited all places listed in Table 1 and measured the corresponding horizontal coordinates. The video record helped also in sorting the quality of individual visual sightings. The trajectory computed was very shallow (near horizontal flight), and in this respect similar to the Peekskill bolide. The body with initial mass of the order of $10^{4} \mathrm{~kg}$ moved with initial velocity of $\approx 15 \mathrm{~km} \mathrm{~s}^{-1}$ and suffered severe gross-fragmentation. Individual larger fragments are well visible on the video record. Geographical coordinates, heights, and velocities of the main body are given for individual time instants during the video recorded part of the trajectory. During this part of the trajectory a model of gross-fragmentation was applied to the observed values. Mass loss at discrete trajectory points was responsible for the majority of the ablation. The orbit of the body was of low eccentricity and low inclination with aphelion close to the Mars orbit. The most probable terminal mass (meteorite mass) of the main body was of about $1 \mathrm{~kg}$. The dark-flight computations are of low accuracy due to the shallow trajectory, and only a large area of some $22 \times 46 \mathrm{~km}$ could be given as possible impact region of meteorites between 0.1 and $10 \mathrm{~kg}$. The relative positions of individual fragments according to the main body during the flight are also given.
\end{abstract}

Key words: meteors, meteoroids

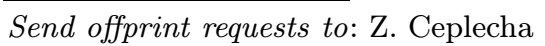

\section{Introduction}

Friday, June 14, 1996, resulted in the hottest day of the year in Galicia (North-Western region of Spain). A temperature of $32^{\circ} \mathrm{C}$ was recorded in Santiago de Compostela the Galicia capital. Because of this many people were in streets, parks, or country houses taking fresh air at late hours. Sky was completely clear (almost New Moon) and both the day and night were of an authentic summertime in spite of not yet being astronomically related to this season of the year. There were almost perfect conditions to see the night sky in its entire splendor.

A bit before the local midnight at $21^{\mathrm{h}} 48^{\mathrm{m}} 20^{\mathrm{s}}$ UTC, an enormous and extremely bright object crossed the sky astonishing and even preoccupying a lot of people who chanced to see the event. A long trajectory and a long time interval during which the object could be seen on the clear sky added more amazement and curiosity. A journalist of a regional newspaper reported that some people in a small village Cacheiras (a suburb of Santiago) being at these late hours near a swimming-pool of their country house have seen an extremely bright light that illuminated the ground all the place round. This was the reason that from its very beginning the event became known publicly as "Cacheiras' UFO". In view of mass-media insistency, and anxious to learn more about the nature of the phenomenon the Astronomical Observatory of the University claimed that much more witnesses (including those from relatively remote locations) and a very careful investigation of the phenomenon had to be carried out in order to determine its properties and the location of the possible impact site.

The collaboration with mass-media (usually interested in "UFO cases") acted like a trigger, and was decisive for obtaining our observational data. We received more than a hundred calls at the Astronomical Observatory "Ramon Maria Aller" during just the first day after the news was 


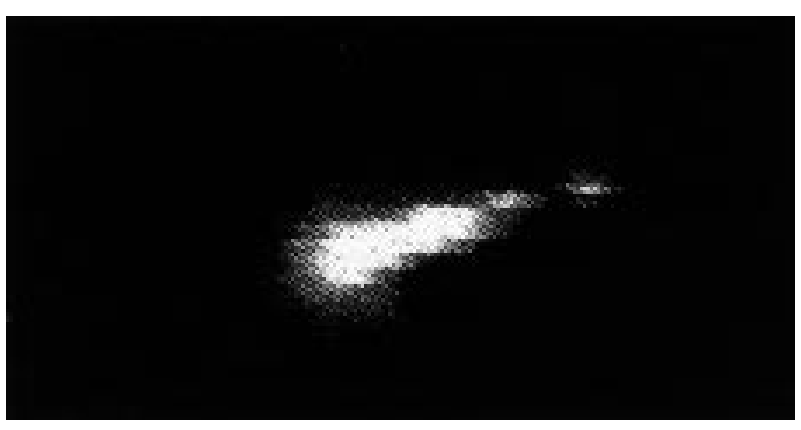

Fig. 1. Video frame No.117 (MET117) shows the bolide appearance at the relative time $4.68 \mathrm{~s}$ with individual fragments 5,6 , and 7 well separated from the main body

released. Among these witnesses there were not only inhabitants of Santiago and its outskirts, but in some cases also people living more than $100 \mathrm{~km}$ away from Santiago. All witnesses described the phenomenon as something they never had seen before. They saw a huge fireball $(\approx$ half of the Moon size) with a striking tail of different colors changing during the flight. It had a tube shape being as bright as full Moon (or even brighter) with a little inclined trail and with angular velocity slow enough to be well observed during many seconds of the flight. However, the most valuable and objective information has been provided by a unique video tape casually recorded during the last $7.6 \mathrm{~s}$ of the bright event.

\section{Video record of the bolide}

The video record was an exceptional opportunity to carry out a detailed study of the event, much better opportunity than if only visual sightings would be available. A group of University students had been celebrating at an evening party on an open terrace at the top of a rather high building $(\approx 30 \mathrm{~m}$ above street level) located near the center of Santiago de Compostela. This terrace is oriented to the East (the bolide moved from SE to N) and the cameraman, J.A. Quiroga just faced the bolide when it started to be enormously bright. He has been recording the dancing party members, but he immediately decided to followup the bright sky phenomenon. Quickly and smoothly he moved the camera up and left to the object, and followed its motion with a great care and ability until the bolide light became definitely extinct. Thus he obtained an exceptionally valuable record of the bolide and its fragmentation. The record owners A.Salazar, D. Neira, and J.A. Quiroga transferred all copyrights of video recorded images to the authors of the present study. One of the frames is presented in Fig. 1 and except the main body shows also 3 larger fragments. The entire available information from video camera is included in this paper in a form of CD ROM copy of the digitized video tape (see Appendix).

\subsection{Compact disk copy of digitized video frames}

With the aim to extract maximal information from video tape, we proceeded to its digitization dividing all record to 191 separate frames recorded on the compact disk (CD) with the following characteristics:

1. Duration: $7.6 \mathrm{~s}$.

2. Resolution on GIF and BMP files: $720 \times 576$ pixels.

3. Resolution on AVI files: $480 \times 360$ pixels.

4. Number of frames: 191 (digitized by 25 frames/s).

5. Digitization code Microsoft Video 1.

6. CD space occupied $34 \mathrm{MB}$.

Since these frames are the essential part of the present work, we consider mandatory to include CD into our paper as an Appendix. Our measurements for the positional reduction of this paper were performed using GIF files.

1. Directory GIFS contains 191 files (三frames) in GIF format.

2. Directory BMPS contains the same frames in BMP format.

3. Directory ANIM contains digitized video in MPG format (MPEG frames) as well as AVI-formated files; they can be reproduced on any PC with Microsoft Windows.

\subsection{Fiducial objects for calibration}

Fortunately, the apparent position of the object during the last part of the recording was low enough for some terrestrial objects to be recorded simultaneously with the bolide. Thus we can make use of these objects as fiducial points for computing horizontal coordinates (azimuth and elevation). Chimneys and TV aerials of some close buildings as well as towers of the famous Cathedral of Santiago de Compostela (the bolide finished the apparent trajectory close to the Cathedral) constitute the fundamental reference points when we assigned the horizontal coordinates to the bolide image at different frames. Once reference points were recognized and established on individual frames, we proceeded to measurements of azimuths and elevations of 10 points using a theodolite installed exactly at the position of the cameraman. Also high quality photographs of the surroundings were taken from the position of the cameraman. They were used for identifying objects on the video frames and also for deriving coordinates of some additional fiducial points.

\section{Visual sightings}

The large number of witnesses made impossible in a few days to visit all locations from where the people called and commented on their sightings. We then decided to make a selection of visual sightings taking into account not only the distances among the locations, but also the quality of 
data and reliability these sightings seem to deserve. Thus we compiled a list of 34 sightings additional to those of the people present at the place, where the video records were taken. Survey of all these sightings is given in Table 1.

We visited all these locations to collect the maximal information, to carry out theodolite coordinate measurements of the beginning and end points of the apparent trail of the bolide, and to gather all data on estimated duration of the event, color of the bolide, on sounds heard etc. Just from the first direct sightings we concluded that the trajectory was almost horizontal and that several fragmentation events were observed in the course of bolide motion. All witnesses but three assured they did not hear any significant sound confirming the absence of any significant noise accompanying this phenomenon.

One has to note some sightings like that one of a person who was walking near a port in Riveira (sighting No.12, in Table 1) at Arousa estuary. Looking from this point (completely clear from obstacles) toward the other side of estuary he was able to observe an ample trail arc of about $100^{\circ}$ and estimated the duration to some $10-12 \mathrm{~s}$. The witnesses from O Barco (No. 21) and Oleiros (No. 28) provided data with directions on the zone maps. A witness from Formaris (No. 26) could compare the event with another luminous object of extreme brightness he observed in January, 1994 (Docobo et al. 1998). He claimed that the 1994 event had a significantly lower brightness. However, this estimate might be influenced by the observational conditions which were rather different in 1994 when the event was seen already at dawn.

\section{Azimuths and zenith distances of the bolide trail from the video record}

We used individual digitized frames for deriving azimuths and elevations of the bolide at different time instants. With reasonable precision, this was possible only for those frames, where fiducial objects of known coordinates were available. There are no stars or other celestial objects in the field of view throughout the whole video record, i.e. from frame 1 to frame 191 (we will refer to frames just only by their numbers: MET001 $\equiv 1$, etc.). Thus we had to limit these computations only to the last part of the trail, where terrestrial objects are present in the field of view of the video camera. Also the beginning part of the recorded trail was calibrated using terrestrial objects in the field of view just before the start of the quick motion of the camera toward the bolide, and following then quantitatively this quick motion. The azimuths and elevations of fiducial points we used in the final calibration are given in Table 2. Their precision is around $\pm 0.1^{\circ}$.

We measured rectangular coordinates of the bolide main body in pixels of the digitized frames. All the frames from 1 to 191 were measured. Only main body is dealt with in this section. Also 13 fragments and the wake
Table 1. Survey of visual sightings arranged in the table as we gradually gathered them. "No" is our reference number of the station; $\lambda, \varphi$, and $H$ are longitude, latitude, and height above sea level of the station; $a_{\mathrm{B}}, h_{\mathrm{B}}$ are azimuth and elevation of the observed first point of the bolide; $a_{\mathrm{E}}, h_{\mathrm{E}}$ are azimuth and elevation of the observed last point of the bolide. Azimuth is the astronomical azimuth $\left(\mathrm{S}=0^{\circ}, \mathrm{W}=90^{\circ}\right)$. Notion $\mathrm{S}$. in the "Station" column means Santiago de Compostela

\begin{tabular}{|c|c|c|c|c|c|c|c|}
\hline No Station & $\lambda_{0}^{\lambda \mathrm{E}}$ & $\varphi_{0}^{\mathrm{N}}$ & $\begin{array}{c}H \\
\mathrm{~km}\end{array}$ & $\underset{\circ}{a_{\mathrm{B}}}$ & $\underset{\circ}{h_{\mathrm{B}}}$ & $\underset{\circ}{a_{\mathrm{E}}}$ & $\begin{array}{c}h_{\mathrm{E}} \\
\circ\end{array}$ \\
\hline 1 S. Hospital & 351.4564 & 42.8850 & 0.26 & 295 & 30 & 210 & 20 \\
\hline 2 S. video & 351.4556 & 42.8764 & 0.28 & (Tal & ble : & 3, Eq. & $\cdot(1))$ \\
\hline 3 S. Pard. & 351.4556 & 42.8758 & 0.28 & 290 & 30 & 210 & 25 \\
\hline 4 S. Lourenzo & 351.4422 & 42.8794 & 0.23 & 265 & 30 & 205 & 20 \\
\hline 5 S. Audit. & 351.4597 & 42.8875 & 0.24 & 305 & 30 & 235 & 30 \\
\hline 6 A Coruna & 351.6000 & 42.3750 & 0.03 & 310 & 35 & 270 & 30 \\
\hline 7 As Neves & 351.5833 & 42.0917 & 0.20 & 250 & 30 & 230 & 30 \\
\hline 8 Ribadavia & 351.8500 & 42.2917 & 0.10 & 250 & 35 & 220 & 30 \\
\hline 9 Montouto & 351.4667 & 42.8417 & 0.30 & 280 & 25 & 210 & 20 \\
\hline 10 Rianxo & 351.1833 & 42.6556 & 0.01 & 230 & 25 & 215 & 20 \\
\hline 11 Covas & 352.3917 & 43.6708 & 0.01 & 0 & 60 & 160 & 45 \\
\hline 12 Riveira & 351.0158 & 42.5583 & 0.01 & 308 & 15 & 212 & 10 \\
\hline 13 Recesende & 351.4444 & 42.8144 & 0.16 & 258 & 25 & 208 & 10 \\
\hline 14 Cacheiras & 351.4472 & 42.8167 & 0.18 & 250 & 30 & 210 & 25 \\
\hline 15 Os Tilos & 351.4611 & 42.8456 & 0.28 & 265 & 30 & 215 & 25 \\
\hline 16 A Bana & 351.2500 & 42.7917 & 0.28 & 310 & 25 & 220 & 25 \\
\hline 17 Guitiriz & 352.1250 & 43.1833 & 0.50 & 285 & 45 & 255 & 45 \\
\hline 18 Dodro & 351.3000 & 42.7167 & 0.02 & 265 & 30 & 210 & 25 \\
\hline 19 Muros & 350.9333 & 42.7750 & 0.01 & 265 & 30 & 225 & 20 \\
\hline 20 Pastoriza & 352.6417 & 43.3333 & 0.55 & 150 & 65 & 142 & 52 \\
\hline $21 \mathrm{O}$ Barco & 353.0000 & 42.4083 & 0.34 & 20 & 35 & 145 & 15 \\
\hline 22 Verin & 352.5667 & 41.9444 & 0.37 & 235 & 60 & 185 & 25 \\
\hline 23 Monterrei & 352.5083 & 41.9389 & 0.46 & 250 & 40 & 180 & 20 \\
\hline 24 Esgos & 352.2833 & 42.3167 & 0.58 & 305 & 50 & 230 & 25 \\
\hline 25 Maceda & 352.3500 & 42.2583 & 0.60 & 220 & 40 & 185 & 10 \\
\hline 26 Formaris & 351.5139 & 42.9222 & 0.29 & 290 & 25 & 200 & 20 \\
\hline 27 Lavacolla & 351.5583 & 42.9083 & 0.32 & 270 & 20 & 200 & 15 \\
\hline 28 Oleiros & 351.6879 & 43.3361 & 0.10 & 280 & 30 & 208 & 25 \\
\hline 29 San P. Nos & 351.6564 & 43.3208 & 0.04 & 245 & 30 & 202 & 25 \\
\hline 30 Lendoiro & 351.7083 & 43.3028 & 0.05 & 235 & 35 & 205 & 30 \\
\hline 31 Bertamir. & 351.3583 & 42.8583 & 0.05 & 300 & 30 & 250 & 25 \\
\hline 32 Brion & 351.3250 & 42.8625 & 0.07 & 290 & 30 & 250 & 25 \\
\hline 33 Rois & 351.3033 & 42.7750 & 0.15 & 270 & 20 & 230 & 20 \\
\hline 34 S. Ma & 351.5667 & 42.7667 & 0.10 & 194 & 30 & 239 & 25 \\
\hline 35 Pobra S.X. & 352.6000 & 42.8500 & 0.40 & 240 & 72 & 168 & 30 \\
\hline
\end{tabular}

phenomenon were measured and results on them are presented in the last section.

\subsection{Calibration of the end part of the video-record}

Rectangular coordinates of the main body were transformed to azimuths, $a$, and elevations, $h$, by using a newly developed least squares version of the old method for positional reductions on wide field images (Ceplecha 1951, 1954a,b). Three objects define this transformation uniquely, but we rather used 4 of them as the lower 
Table 2. Azimuths and elevations of fiducial points used for calibration of the video frames

\begin{tabular}{|c|c|c|}
\hline object & azimuth & elevation \\
\hline A chimney & $186.37^{\circ}$ & $7.09^{\circ}$ \\
\hline B cathedral & 192.18 & 3.03 \\
\hline C chimney & 206.11 & 3.84 \\
\hline D chimney & 213.66 & 4.02 \\
\hline E chimney & 221.58 & 4.41 \\
\hline F chimney & 263.83 & 5.45 \\
\hline G TV aerial & 277.83 & 8.62 \\
\hline H TV aerial & $317.93^{\circ}$ & $9.65^{\circ}$ \\
\hline
\end{tabular}

Table 3. Azimuths and elevations of the bolide apparent trail from the video frames

\begin{tabular}{lll}
\hline frame no. & azimuth & elevation \\
\hline 150 & $204.2^{\circ} \pm 0.3^{\circ}$ & $22.5^{\circ} \pm 0.3^{\circ}$ \\
155 & $202.9^{\circ} \pm 0.3^{\circ}$ & $21.9^{\circ} \pm 0.3^{\circ}$ \\
160 & $201.7^{\circ} \pm 0.3^{\circ}$ & $21.3^{\circ} \pm 0.3^{\circ}$ \\
165 & $200.6^{\circ} \pm 0.3^{\circ}$ & $20.6^{\circ} \pm 0.3^{\circ}$ \\
170 & $199.3^{\circ} \pm 0.3^{\circ}$ & $20.4^{\circ} \pm 0.3^{\circ}$ \\
175 & $198.2^{\circ} \pm 0.5^{\circ}$ & $19.8^{\circ} \pm 0.5^{\circ}$ \\
\hline
\end{tabular}

limit, just to have an additional check. This seems well grounded, because terrestrial objects on the video record are visible only marginally being very close to the sensitivity limit of the camera, and thus hard to measure. This way only the frames from 147 to 176 were reduced, all with fiducial objects A, B, D, E, and some with additional object $\mathrm{C}$. The resulting azimuths and elevations are given in Table 3 with their standard deviations. They were derived for "average" points (i.e. smoothed over 5 frames centered at the given frame). We used only these 6 positions $(a, h)$ in Table 3 to define the great circle of the apparent trail of the bolide from the video frames. The resulting great circle is given by Eq. (1).

$\sin (164.052-a)=-1.56256 \tan (h)$.

Standard deviation of one position in Table 3 from the average apparent trail given by Eq. (1) is $\pm 0.16^{\circ}$. Using the great circle defined by Eq. (1) and the measured position of the body in frame 191 (the last record of the body), we can derive the terminal point of the video recorded trail as $a=195.50^{\circ}$ and $h=18.46^{\circ}$.

\subsection{Calibration of the beginning part of the video-record}

The cameraman documented a students party, which took place at a wide open balcony with clear skies overhead. When he saw the bolide, he quickly and smoothly moved the camera onto the bolide and followed its motion also quite smoothly until the bolide ceased to be visible (frame 191). The camera was still in motion during the exposure time of frame 1 , while frame 2 is the first with almost no smear effect, the bolide image being already nicely

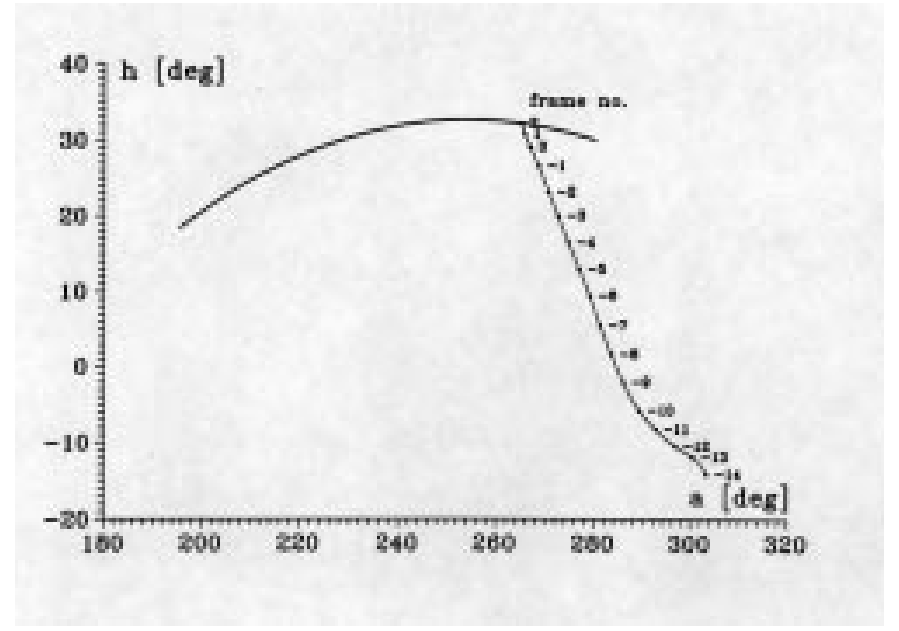

Fig. 2. Quick motion of the video camera from terrestrial objects (frame -14) to the bolide frame 2; $a$ is the azimuth and $h$ the elevation. Thick line is the bolide apparent trail as given by Eq. (1); thin line with numbered points is the camera motion; the points correspond to the position of the video camera at individual frames during the quick motion

pointed. We have chosen position of the bolide in frame 2 and projected it (computational way) onto the frame with scenery of the student party just one frame before the camera started to move quickly to the bolide: in our system of frame numbers, this was frame no. -14 . Fiducial objects $F$, $\mathrm{G}, \mathrm{H}$ were used to derive this starting position of the camera as $a=303.3^{\circ}, h=-14.1^{\circ}$, using the same method as in previous section. We measured then differences proportional to the differences in azimuth and elevation, from a frame to the next frame, $\Delta a, \Delta h$, starting from frame -14 to frame -13 , from frame -13 to frame -12 , and so on until frame 2 . These differences were measured relatively to terrestrial objects on frames -14 to -8 , and relatively to the bolide itself on frames -4 to 2 ; frames $-7,-6$, and -5 , where there were no objects to compare with, were interpolated. Having the great circle of the apparent trail as given by Eq. (1), and starting at $a=303.3^{\circ}, h=-14.1^{\circ}$, we added all the differences $\Delta a, \Delta h$, and we had to match this great circle just at frame 2 . This condition defined the proportionality constant, and so defined also the position of the bolide on frame 2 as $a=264.96^{\circ}, h=32.15^{\circ}$. The derived motion of the camera is given in Fig. 2, where also the position of the great circle of the trajectory as defined by Eq. (1) is given.

\section{Atmospheric trajectory from the video-record combined with visual observations}

The location of the video camera and the apparent trail given by Eq. (1) defines a plane, in which the atmospheric trajectory had to be located. Visual sightings of casual observers were necessary to derive the atmospheric trajectory in this plane. We have used a method and 
computer code by Borovička (1990) to do this. Each visual observation can be checked not only against the others, but against the video recorded apparent trail, which makes the decision on what visual observations are realistic more objective. In the final reduction we have used the following visual observations of Table 1 combined with the video recorded trail from station 2 : stations $1,3,4,5,6,7,8,9,10$, $12,14,15,16,18,19,21,24,26,31,32$, and 33 .

The first observed point from station 12 was located at

$\lambda=352.94^{\circ} \pm 0.05^{\circ} \mathrm{E}, \varphi=41.43^{\circ} \pm 0.03 \mathrm{~N}$,

$h=52.4 \pm 3.2 \mathrm{~km}$.

Video recorded frame 2 was located at

$\lambda=352.20^{\circ} \pm 0.04^{\circ} \mathrm{E}, \varphi=42.92^{\circ} \pm 0.03 \mathrm{~N}$,

$h=38.9 \pm 3.0 \mathrm{~km}$.

The last point observed (video recorded frame 191) was located at

$\lambda=351.78^{\circ} \pm 0.04^{\circ} \mathrm{E}, \varphi=43.72^{\circ} \pm 0.03 \mathrm{~N}$,

$h=34.0 \pm 2.9 \mathrm{~km}$.

Azimuth and zenith distance of the radiant for this terminal point (frame 191) were

$a_{\mathrm{R}}=339.0^{\circ} \pm 1.2^{\circ}, z_{\mathrm{R}}=87.3^{\circ} \pm 1.5^{\circ}$,

and the right ascension and declination of the apparent radiant were

$\alpha_{\mathrm{R}}=250.1^{\circ} \pm 1.5^{\circ}, \delta_{\mathrm{R}}=-39.9^{\circ} \pm 1.5^{\circ}$.

The trajectory was very shallow, almost tangential to the Earth's surface. The Earth-grazing character of the trajectory is also the main reason that there was enough time to take the video record of the bolide. On the other hand, the standard deviation of distances along the trajectory are rather high, i.e. $\pm 5.3 \mathrm{~km}$ for one point. Thus also the velocities derived directly from the end part of the trajectory using distances of only about $20 \mathrm{~km}$ are inaccurate. This will be dealt with in the next section. Because the final data on velocity and thus also for location of individual video frames can be improved by using combination of the geometrical solution of this section. with modeling the motion, ablation (including discrete fragmentation), and luminosity of the bolide, we present also the final data on locations together with velocities in Table 4 of the next section.

\section{Velocity and theoretical modeling}

The frequency of the video frames corresponds to PAL system with 25 frames per second. Thus neighboring frames are separated by $0.04 \mathrm{~s}$. We will use a relative time defined as $t=n / 25$ in seconds, where $n$ is the already defined frame number. If we use the end portion of the trajectory (frames 150 to $191 ; t=6.0 \mathrm{~s}$ to $7.64 \mathrm{~s}$ ), velocity $v=13 \pm 5 \mathrm{~km} \mathrm{~s}^{-1}$ for $t=6.8 \mathrm{~s}$ is resulting. This means that there is not much useful information on velocity during the end portion of the trajectory. This situation changes when we compute the average velocity from frame 2 to frame 175 (from $t=0.08 \mathrm{~s}$ to $t=7.0 \mathrm{~s}$ ). The resulting average velocity is $v=12.8 \pm 1.1 \mathrm{~km} \mathrm{~s}^{-1}$ for

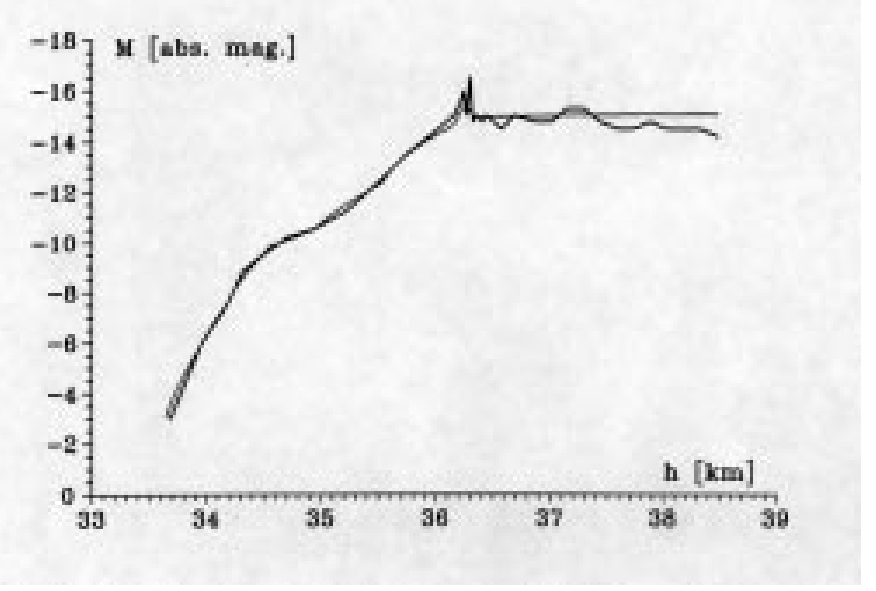

Fig. 3. Light curve of the bolide. Absolute magnitudes are plotted against heights. Thick line corresponds to the video camera observation; thin line corresponds to the model derived magnitudes

$t=3.5 \mathrm{~s}$. In computing the following model of motion, ablation, and radiation, our first constraint is keeping this value of velocity. The second constraint is keeping the observed heights and locations derived geometrically in the preceding section. And the third constraint is copying the observed brightness of the bolide as close as possible.

We performed only a schematic photometry using sizes of the image in the video frames transformed to absolute stellar magnitudes (distance $100 \mathrm{~km}$ ). We assumed that the part of the steady brightness before the maximum at frame 90 was of about apparent magnitude -15 . We also assumed that the limit of the camera recording was at about magnitude -3 . The resulting observed light curve is compared in Fig. 3 with the modeled light curve.

Our model is based on gross-fragmentation model (Ceplecha et al. 1993) for computing the motion and ablation of the main body, and on classical luminous equation, $I=-\left(\tau v^{2} / 2\right) \mathrm{d} m / \mathrm{d} t$, modified for time elapsed from fragmentation point to the instant when the fragments stopped to contribute to luminosity, $I$, of the main body (they are either visible as separate objects or already not radiating enough to be detected). The luminous efficiency, $\tau$, we used takes care of the new calibration derived from the Lost City bolide and meteorite fall (Ceplecha 1996). Luminous efficiency was taken as a function of mass, $m$, in our model: for masses below $10 \mathrm{~g}$ luminous efficiency is given by the experimental values derived by Ayers et al. (1970), and for masses over $100 \mathrm{~kg}$ luminous efficiency is given by the new Lost City calibration. For masses between these two values, luminous efficiency was computed by linear interpolation in logarithms (corresponding to linear interpolation in stellar magnitudes). The velocity dependence of luminous efficiency was kept the same as given by Ceplecha \& McCrosky (1976, Table 1).

In modeling our case we assumed ablation coefficient $\sigma=0.014 \mathrm{~s}^{2} \mathrm{~km}^{-2}$, the average observed value for type I 
Table 4. Trajectory of the bolide: geographical coordinates, heights and velocities for the main body at individual video frames; standard deviations of velocities from frame 1 to frame 191 are about $10 \%$ of their values

\begin{tabular}{|c|c|c|c|c|c|}
\hline frame & $\bar{t}$ & $\lambda$ & $\varphi$ & $h$ & $v$ \\
\hline No. & $\mathrm{s}$ & ${ }^{\circ} \mathrm{E}$ & ${ }^{\circ} \mathrm{N}$ & $\mathrm{km}$ & $\mathrm{km} \mathrm{s}^{-1}$ \\
\hline beg. & $(-12.37)$ & 352.94 & 41.43 & 52.4 & 14.9 \\
\hline 1 & 0.04 & 352.20 & 42.91 & 38.9 & 13.5 \\
\hline 10 & 0.40 & 352.18 & 42.95 & 38.6 & 13.4 \\
\hline 20 & 0.80 & 352.16 & 42.99 & 38.3 & 13.3 \\
\hline 30 & 1.20 & 352.13 & 43.04 & 37.9 & 13.2 \\
\hline 40 & 1.60 & 352.11 & 43.08 & 37.6 & 13.1 \\
\hline 50 & 2.00 & 352.09 & 43.12 & 37.3 & 13.0 \\
\hline 60 & 2.40 & 352.07 & 43.16 & 37.0 & 12.9 \\
\hline 70 & 2.80 & 352.05 & 43.21 & 36.7 & 12.8 \\
\hline 80 & 3.20 & 352.02 & 43.25 & 36.4 & 12.7 \\
\hline 90 & 3.60 & 352.00 & 43.29 & 36.2 & 12.6 \\
\hline 100 & 4.00 & 351.98 & 43.33 & 35.9 & 12.3 \\
\hline 110 & 4.40 & 351.96 & 43.38 & 35.6 & 11.9 \\
\hline 120 & 4.80 & 351.94 & 43.42 & 35.4 & 11.5 \\
\hline 130 & 5.20 & 351.91 & 43.46 & 35.2 & 11.1 \\
\hline 140 & 5.60 & 351.89 & 43.50 & 35.0 & 10.7 \\
\hline 150 & 6.00 & 351.87 & 43.55 & 34.8 & 10.3 \\
\hline 160 & 6.40 & 351.85 & 43.59 & 34.6 & 9.8 \\
\hline 170 & 6.80 & 351.83 & 43.63 & 34.4 & 9.2 \\
\hline 180 & 7.20 & 351.80 & 43.67 & 34.2 & 8.6 \\
\hline 191 & 7.64 & 351.78 & 43.72 & 34.0 & 7.5 \\
\hline end & (11.76) & 351.70 & 43.87 & 33.1 & 3.0 \\
\hline
\end{tabular}

bolides (stone). Any attempt to use larger values was unsuccessful because it was not possible to bring the body so deep into the atmosphere with such a grazing trajectory and to keep it moving with enough mass for such a long time interval. We then assumed different initial masses, initial velocities, location of fragmentation points, and amounts of fragmented mass so that we kept the observed heights and distances along the trajectory, and luminosity as observed. The resulting values of velocities are given in Table 4, where time, $t$, is coordinated to individual video frames, and the geographical coordinates, $\lambda, \varphi$, and heights, $h$, are those derived in the previous Sect. by combination of the video record with the visual observations from other locations. Observed heights and observed geographical coordinates of the bolide at instants of individual frames well correspond to model computed velocities, $v$, and to the average velocity derived directly from observed distances $\left(12.8 \pm 1.1 \mathrm{~km} \mathrm{~s}^{-1}\right)$. The initial velocity (outside the atmosphere), $v_{\infty}$, corresponding to values in Table 4 resulted as

$v_{\infty}=15.1 \pm 1.4 \mathrm{~km} \mathrm{~s}^{-1}$.

The initial mass (before ablation and fragmentation started), $m_{\infty}$, and mass, $m_{\mathrm{B}}$, at the start of the video recording (frame 2) resulted as

$m_{\infty}=9100 \mathrm{~kg}$ and $m_{\mathrm{B}}=6500 \mathrm{~kg}$.

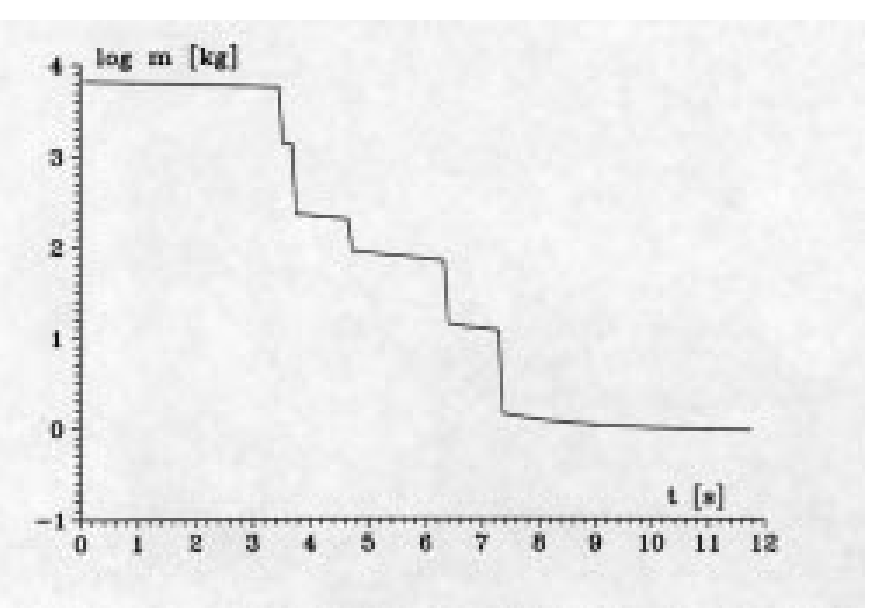

Fig. 4. Mass of the main body as resulted from the model is plotted against time

The fragmentation history can be seen in Fig. 4 where logarithm of mass of the main body is plotted as a function of time. The sudden changes in mass of the main body are just the resulting fragmentation points. They also correspond to releasing of individual larger fragments seen behind the main body on the video frames. Maximum dynamic pressure the body encountered during the modeled entry was equal to $1 \mathrm{MPa}$ and was achieved just at the point of maximum brightness, where the first larger grossfragmentation happened. We were not able to model an early fragmentation before the maximum light and above $37 \mathrm{~km}$ height, but this should form only a small correction to the initial mass, which might be larger by about $10 \%$ than the above given value.

The point denoted "end" in Table 4 was computed as extrapolation after frame 191 down to $3 \mathrm{~km} \mathrm{~s}^{-1}$ (velocity at which ablation stops) keeping the same value for the ablation coefficient. This computation also yielded the value of the terminal mass as $0.94 \mathrm{~kg}$. Thus one would expect meteorite falls of kilogram masses. The upper limit of a possible meteorite should not exceed $10 \mathrm{~kg}$.

We also applied a single body model to all the resulting values of Table 4 in order to check our model by such a "first approximation". This way we received also an "average" apparent ablation coefficient $\sigma=0.11 \mathrm{~s}^{2} \mathrm{~km}^{-2}$ documenting the high degree of fragmentation of the meteoroid. This value is quite similar to the value of Peekskill's bolide and meteorite fall (Brown et al. 1994; Ceplecha et al. 1996) derived also from a single body model. The SP960614 bolide resembles the Peekskill bolide also by its Earth grazing trajectory.

\section{Orbit}

The initial velocity and apparent radiant were used to compute the orbit following procedures described by Ceplecha (1987). The orbit is presented in Table 5 and is 
Table 5. Radiant and orbit; angular elements referred to J2000

\begin{tabular}{llcll}
\hline & & & & \\
$v_{\infty}$ & $\mathrm{km} \mathrm{s}^{-1}$ & 15.1 & \pm & 1.4 \\
$\alpha_{\mathrm{R}}$ & degrees & 250.1 & \pm & 1.5 \\
$\delta_{\mathrm{R}}$ & degrees & -39.9 & \pm & 1.5 \\
$v_{\mathrm{G}}$ & $\mathrm{km} \mathrm{s}^{-1}$ & 10.0 & \pm & 2.1 \\
$\alpha_{\mathrm{G}}$ & degrees & 262. & \pm & 6. \\
$\delta_{\mathrm{G}}$ & degrees & -60. & \pm & 5. \\
$v_{\mathrm{H}}$ & $\mathrm{km} \mathrm{s}^{-1}$ & 30.9 & \pm & 1.2 \\
$\varepsilon_{\mathrm{G}}$ & degrees & 89. & \pm & 3. \\
$\varepsilon_{\mathrm{H}}$ & degrees & 161. & \pm & 3. \\
$a$ & A.U. & 1.12 & \pm & 0.10 \\
$e$ & & 0.27 & \pm & 0.08 \\
$q$ & A.U. & 0.816 & \pm & 0.022 \\
$Q$ & A.U. & 1.42 & \pm & 0.22 \\
$\omega$ & degrees & 86. & \pm & 8. \\
$\Omega$ & degrees & 264.1443 & \pm & 0.0002 \\
$i$ & degrees & 11.5 & \pm & 1.0 \\
\hline
\end{tabular}

$v_{\infty}$ initial velocity,

$\alpha_{\mathrm{R}}, \delta_{\mathrm{R}}$ right ascension and declination of observed radiant, $v_{\mathrm{G}}$ geocentric velocity,

$\alpha_{\mathrm{G}}, \delta_{\mathrm{G}}$ right ascension and declination of geocentric radiant, $v_{\mathrm{H}}$ heliocentric velocity,

$\varepsilon_{\mathrm{G}}, \varepsilon_{\mathrm{H}}$ elongation of $\mathrm{H}$ and $\mathrm{G}$ radiant from Earth's apex, standard notation for orbital elements is used.

of low eccentricity and low inclination with aphelion close to the Mars orbit.

\section{Dark flight}

The Earth's grazing trajectory is extremely unfavorable for computing dark-flight distances and for predicting of an impact area of meteorites. Thus we limited us only to rather schematic computations without wind field being included. The computation of the dark-flight started at point denoted "end" in Table 4, at which the azimuth of the radiant (instantaneous motion and horizon) was $339.02^{\circ}$ and the zenith distance of the radiant was $86.94^{\circ}$. The equations of motion are those given in Ceplecha et al. (1998), pages 320-322. The results are given in Table 6 for three masses representing a mass range of possible meteorites. The many tens of kilometers long dark-flight distances and the uncertainties of the trajectory slope define a very large impact area, which is given as being $11 \mathrm{~km}$ to both sides of the line in Table 6 . Thus the predicted impact area is a $22 \mathrm{~km}$ wide and about $46 \mathrm{~km}$ long stripe symmetrically to the line of Table 6 .

\section{Fragmentation}

Throughout the entire video-recorded trail, the main body has been the brightest and the leading. The preceding sections dealt with the main body. However, in addition to the main body, numerous smaller fragments and a wake
Table 6. Geographical coordinates of impact points for different masses, $m_{\mathrm{E}}$. Notation: $L$ is the horizontal dark-flight distance starting from point "end", $T$ is the time interval from point "end" to the Earth's surface, and $v_{\mathrm{s}}$ is the impact velocity

\begin{tabular}{cccccc}
\hline$m_{\mathrm{E}}$ & $\begin{array}{c}\lambda \\
\mathrm{kg}\end{array}$ & $\begin{array}{c}\varphi \\
\text { degrees E }\end{array}$ & $\begin{array}{c}L \\
\text { degrees } \mathrm{N}\end{array}$ & $\begin{array}{c}T \\
\mathrm{~km}\end{array}$ & $\begin{array}{c}v_{\mathrm{s}} \\
\mathrm{m} \mathrm{s}^{-1}\end{array}$ \\
\hline 0.1 & 351.60 & 44.06 & 23 & 400 & 38 \\
1. & 351.54 & 44.18 & 37 & 290 & 56 \\
10. & 351.45 & 44.35 & 57 & 215 & 82 \\
\hline
\end{tabular}

Standard deviation in $\lambda= \pm 0.10^{\circ}( \pm 8 \mathrm{~km})$, standard deviation in $\varphi= \pm 0.07^{\circ}( \pm 8 \mathrm{~km})$.

are visible on the video record. We were able to measure positions of 13 individual fragments and positions of the points of the wake termination, all relatively to the main body. The instantaneous direction of motion of the main body and its distance from the video-camera derived in the preceding sections were used to transform the measured angular distances into metric distances. The results are given in Figs. 5 and 6.

The total metric distance $L$ of a fragment (or the terminal point of the wake) from the main body can be expressed by means of the following 3 components: the one along the trajectory, $l$, is the distance $L$ projected perpendicularly onto the main body trajectory (counted positively behind the main body); the two components lateral to the trajectory can be expressed as $d$, the distance $L$ projected perpendicularly onto the line which is perpendicular to both the trajectory and the instantaneous vision line (the + sign means that the point is above the trajectory, and the - sign means below the trajectory), and the other one, $p$, the distance $L$ projected perpendicularly onto the vision line. Because the information on the individual angular distances of fragments from the main body is available from only the one video station, we do not know the lateral component $p$. On the other hand, dynamical reasons made $l$ much larger than both $d$ and $p$ (the observed $d$ does not exceed $0.3 \mathrm{~km}$, while $l$ are as large as $6 \mathrm{~km}$. There is no reason to assume that the two lateral distances $p$ and $d$ would be too much different. If $p$ and $d$ do not exceed $10 \%$ of $l$ (which is the case for almost all measured values), the difference $L-l$ does not exceed $3 \%$, a negligible difference in scope of all the other uncertainties. Thus the distance $l$ behind the main body is very close to the total metric distance $L$.

The fragments separated at different time instants (Fig. 5). Fragments 1 and 2 separated somewhere close to $t \doteq 1 \mathrm{~s}$ : this early fragmentation was not included into the theoretical model described in the preceding section, because it was not visible on the light curve (except that the whole small increase of brightness before $t=2 \mathrm{~s}$ might be related to fragment 1). Thus the initial mass might be somewhat (by $\approx 10 \%$ ) greater than derived in the section on theoretical modeling. Also the first part of the wake, which seems to be mostly gaseous, could contain 


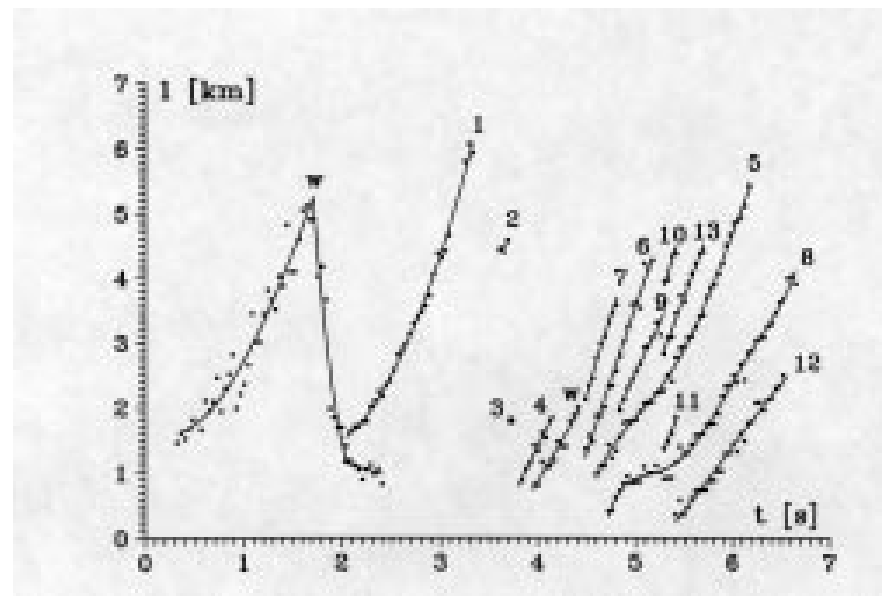

Fig. 5. Distance $l$ of individual fragments behind the main body is plotted against time $t=n / 25$, where $n$ is the frame number. Fragments are numbered. The symbol "w" is used for the wake and means the terminal point of the wake behind the main body. Points represent direct measurements on individual frames; lines fitting these points represent a smooth change of $l$ for the individual fragments. Fragment 3 was observed only on one frame. The wake consists of two parts, before and after the main fragmentation, which took place between 3.5 and $3.7 \mathrm{~s}$

small fragments from this early fragmentation; this part of wake reached the maximum distance behind the main body of more than $5 \mathrm{~km}$ at $t=1.7 \mathrm{~s}$, and then quickly shortened to values below $1 \mathrm{~km}$. Fragments 3, 4, 5, 6, 7, 9,10 , and 13 separated from the main body during atleast-two closely-separated events between $t=3.5$ and $4 \mathrm{~s}$. This corresponds to the main double maximum on the light curve at $t=3.6$ and $3.7 \mathrm{~s}$. Fragments 9, 10, 13 may be secondary fragments originating from fragment 5 . Fragment 8 separated from the main body at $t \doteq 4.6 \mathrm{~s}$. Fragment 11 is a secondary one separated from fragment 8 . Small fragment 12 separated at $t \doteq 5.3 \mathrm{~s}$; its significance for the theoretical model of mass-loss of the main body is negligible.

Thus fragments 3 to 13 (except 12) originated from 3 major break-up events with the largest mass-loss rate $(\mathrm{d} m / \mathrm{d} t)$ as is evident from Fig. 4. Later break-ups after $t=5.5 \mathrm{~s}$ (at least two major events more) did not show individual fragment trails: it is demonstrated only by a very slow brightness decay after the main maximum and by a bump on the light curve between $t=6$ and $7 \mathrm{~s}$. Theoretical mass-loss rate in Fig. 4 between 5 and $8 \mathrm{~s}$ is $3 \times$ less than between 3.5 and $5 \mathrm{~s}$. Fragments released after $t=5.5 \mathrm{~s}$ were already so small that they contributed only to the light of the main body collectively (evaporating dust cloud), and were invisible (below the sensitivity limit) when they traveled to distances, where their images could be separated from the main body. The release time (from break-up to visibility) was about $0.5 \mathrm{~s}$ for all break-ups. This value proved also to be the best for the theoretical

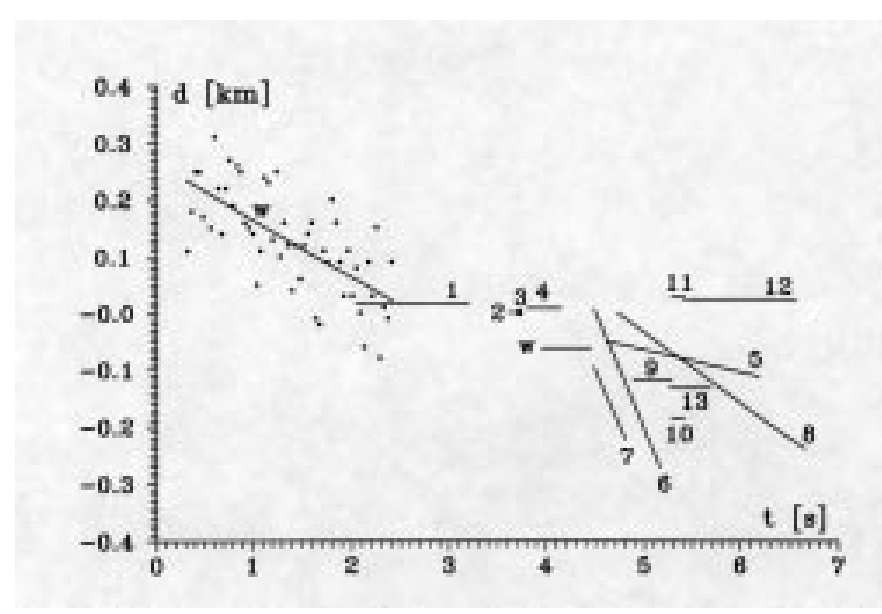

Fig. 6. Lateral distance $d$ of individual fragments above $(+)$ and below $(-)$ the trajectory is plotted against time. They represent the minimum lateral distance possible. Points representing measurements on individual frames are given only for the first part of the wake. They show a typical spread of our measurements. Only the average smooth lines are given for all fragments and the second part of the wake " $w$ " (plotting points of individual measurements would make the figure uncomprehensible). The spread of the points for individual fragments is of the same order as the total change of $d$ over the corresponding time interval; thus only average values of $d$ are available for most of the fragments

modeling of the light curve and was also in agreement with first visibilities of individual fragments (Fig. 5).

Lateral distances $d$ shown in Fig. 6 are small values if compared to $l$. This means that spread of the $d$-values for any fragment measured at individual frames is relatively large and only average values or linear trends could have been determined. Most of the fragments were close to the trajectory or by most of $0.3 \mathrm{~km}$ below it. The initial wake termination started more than $0.2 \mathrm{~km}$ above the trajectory and moved then just onto the trajectory. Small fragment 2 is an exception: this was the only one with rather quick lateral motion; the average value in Fig. 6 originates from three consecutive values: $3.6 \mathrm{~s}, 0.6 \mathrm{~km}$ above the trajectory; $3.64 \mathrm{~s}$ at the trajectory; $3.68 \mathrm{~s}, 0.6 \mathrm{~km}$ below the trajectory.

Acknowledgements. Our sincere thanks are due to Dr. V. Tamazian from Astronomical Observatory "R.M. Aller" for his helpful comments, to Mr. J. Ramirez from Supercomputers Center of Galicia for his special work in video tape digitization. We are very much obliged to the authors of the video record of the bolide, Mr. A. Salazar, Mr. D. Neira, and Mr. J.A. Quiroga for their kind collaboration. Our special thanks are appointed to the Investigation Vice-Rectorate of the University of Santiago de Compostela for their financial support. We are pleased to announce that this work has also been supported by Contract AJ-4706 of the Sandia National Laboratories, and by the Grant Agency of the Czech Republic (205/97/0700). 


\section{Appendix A: Digitized video frames on CD}

The attached CD ROM contains the digitized video tape images. Directories "GIFS" and "BMPS" contain 191 files each in the formats .GIF and .BMP respectively. The video record total duration is $7.6 \mathrm{~s}$ and time elapsed between two consecutive frames (files) is $0.04 \mathrm{~s}$. Directory "ANIM" contains digitized video tape in .AVI format and can be reproduced with any PC using Windows NT and MPEG (MPG).

\section{References}

Ayers W.G., McCrosky R.E., Shao C.-Y., 1970, Smithsonian Astrophys. Obs. Spec. Rep. 317, 1

Borovička J., 1990, Bull. Astron. Inst. Czechosl. 41, 391
Brown P., Ceplecha Z., Hawkes R.L., Wetherill G., Beech M., Mossman K., 1994, Nat 367, 624

Ceplecha Z., 1951, Bull. Astron. Inst. Czechosl. 2, 105

Ceplecha Z., 1954a, Bull. Astron. Inst. Czechosl. 5, 9

Ceplecha Z., 1954b, Bull. Astron. Inst. Czechosl. 5, 21

Ceplecha Z., 1987, Bull. Astron. Inst. Czechosl. 38, 222

Ceplecha Z., 1996, A\&A 311, 329

Ceplecha Z., Borovička J., Elford W.G., ReVelle D.O., Hawkes R.L., Porubčan V., Šimek M., 1998, Space Sci. Rev. 84, 327

Ceplecha Z., Brown P., Hawkes R.L., Wetherill G., Beech M., Mossman K., 1996, Earth, Moon, and Planets 72, 395

Ceplecha Z., McCrosky R.E., 1976, J. Geophys. Res. 81, 6257

Ceplecha Z., Spurný P., Borovička J., Keclíková J., 1993, A\&A 279,615

Docobo J.A., Spalding R.E., Ceplecha Z., Diaz-Fierros F., Tamazian V.S., Onda Y., 1998, Meteoritics \& Planetary Science 33, 57 